\title{
Hepatitis B virus $X$ protein upregulates DNA methyltransferase 3A/3B and enhances SOCS-1CpG island methylation
}

\author{
XIAOYU FU ${ }^{1}$, XIAOLING SONG ${ }^{2}$, YANYAN LI ${ }^{3}$, DEMING TAN $^{1}$ and GUOZHEN LIU ${ }^{1}$ \\ ${ }^{1}$ Department of Infectious Disease, Key Laboratory of Viral Hepatitis of Hunan, Xiangya Hospital, Central South University, \\ Changsha, Hunan 410008; ${ }^{2}$ Department of Infectious Disease, East of People's Hospital, Linyi, Shandong 276034; \\ ${ }^{3}$ Department of Infectious Disease, The Eighth People's Hospital of Nanchang, Nanchang, Jiangxi 330008, P.R. China
}

Received December 26, 2014; Accepted September 22, 2015

DOI: $10.3892 / \mathrm{mmr} .2015 .4545$

\begin{abstract}
The aim of the present study was to investigate the effect of hepatitis B virus $\mathrm{X}$ protein (HBx) on the expression of DNA methyltransferase (DNMT)3A/3B and suppressors of cytokine signaling-1 (SOCS-1), as well as promoter $\mathrm{CpG}$ island methylation of the SOCS-1 gene. Stable hepatocyte cell lines expressing the HBx gene (pcDNA-X/QSG7701) or an empty gene (pcDNA3.0/QSG7701) were established. Reverse transcription quantitative polymerase chain reaction (PCR) was used to detect the mRNA expression levels of DNMT3A/3B and SOCS-1. Immunohistochemistry was used to detect the protein expression of DNMT3A/3B. Methylation-specific PCR (MSP) was used to detect the methylation status of the SOCS-1 gene promoter. The mRNA and protein expression levels of DNMT3A/3B were significantly higher in the pcDNA-X/QSG7701-transfected cells, compared with those in the pcDNA3.0/QSG7701 or non-transfected QSG7701 cells $(\mathrm{P}<0.05)$, whereas the relative mRNA expression of SOCS-1 was significantly lower in the pcDNA-X/QSG7701 cells compared with the pcDNA3.0/QSG7701 and non-transfected QSG7701 cells $(\mathrm{F}=19.6 ; \mathrm{P}<0.05)$. Western blot analysis showed that the protein expression of SOCS-1 was significantly lower in the pcDNA-X/QSG7701 cells, compared with the pcDNA3.0/QSG7701 or non-transfected QSG7701 cells $(\mathrm{F}=19.4 ; \mathrm{P}<0.05)$. The results of the MSP analysis showed that SOCS-1 promoter region methylation was present only in the pcDNA-X/QSG7701 cells. The HBV-X gene upregulated the mRNA and protein expression levels of DNMT3A/3B, downregulated the expression of SOCS-1 and increased SOCS-1 gene promoter CpG island methylation. This may
\end{abstract}

Correspondence to: Professor Guozhen Liu, Department of Infectious Disease, Key Laboratory of Viral Hepatitis of Hunan, Xiangya Hospital, Central South University, 87 Xiangya Road, Changsha, Hunan 410008, P.R. China

E-mail: 35521389@qq.com

Key words: DNA methylation transferase 3A/3B, hepatitis B virus, hepatitis B virus X protein, hepatoma cells, inhibition of cytokine signal transduction molecule-1, methylation provide a potential explanation of the mechanism underlying HBx-associated hepatocellular carcinoma.

\section{Introduction}

Hepatocellular carcinoma (HCC), a common malignant tumor with a mortality rate of thousands of individuals annually, represents $45 \%$ of liver cancer-associated mortality in China. Numerous epidemiological surveys have suggested that chronic hepatitis B viral (HBV) infection is a major cause of primary liver cancer, and $>80 \%$ of patients with liver cancer have been reported to have HBV infection (1), suggesting that HBV infection is closely associated with HCC. Increasing evidence suggests that the HBV X protein (HBx) is important in HCC pathogenesis, however, the specific mechanism underlying $\mathrm{HBx}$-mediated carcinogenesis remains to be elucidated. HBx has been widely recognized as a carcinogenic agent, which has global effects on host cell genes, and the integration of HBV DNA into the host genome, inactivation of tumor suppressor genes and activation of oncogenes are considered to be closely associated with HCC development (2). In addition to gene mutations and loss of chromosomal materials, tumor suppressor gene promoter hypermethylation is a mechanism, and often the only mechanism, for tumor suppressor gene inactivation (3). Previous studies have indicated that HBx has a unique effect of transactivation, which directly or indirectly interacts with proteins and, thus, is involved in infected cell signal transduction, apoptosis and cell cycle regulation (1). Previously, the HBx protein has been shown to induce epigenetic silencing of genes, which are potential predisposing factors for HCC, and is associated with inactivation of the hypermethylation of HCC tumor suppressor genes (4).

SOCS-1 protein is a negative regulator of the Janus kinase (JAK)/signal transducer and activator of transcription (STAT) signaling pathway, and the SOCS-1 gene is a potential tumor suppressor (3). However, whether HBx affects the expression of SOCS-1, and whether it may be a possible mechanism for $\mathrm{HBx}$-induced $\mathrm{HCC}$ remains to be elucidated. Therefore, the present study investigated the effects of HBx on the gene expression of SOCS-1 at transcriptional and translational levels, and examined the potential mechanism underlying HBx-induced HCC development. 
At present, several studies have suggested that tumor suppressor gene hypermethylation is present in lung cancer, hematological malignancies, and other types of cancer (5-9). Oncogene hypomethylation and tumor suppressor gene hypermethylation are important mechanisms for tumor development. DNA methylation, via DNA methyltransferase (DNMT), receives a methyl group on the cytosine of the $\mathrm{CpG}$ DNA dinucleotide, forming 5-methylcytosine. The DNA methyltransferase family includes DNMT1, DNMT3A and DNMT3B, of which DNMT1 is responsible for maintaining methylation, and acts on the semi-methylated substrates that are present in almost all somatic cells. DNMT1 is expressed at high levels in proliferating cells, and DNMT3A and DNMT3B are considered to be involved in de novo DNA methylation, being expressed at high levels in the embryonic stem cells and early embryos. The expression levels of DNMT3A and DNMT3B in normal somatic cells are relatively low, however, they are expressed at high levels in cancer cells (10). Therefore, the present study also investigated the effects of $H B x$ on the expression of DNA methyltransferase $3 \mathrm{~A} / 3 \mathrm{~B}$ in cells, and examined the molecular mechanism underlying $H B x$-induced HCC.

\section{Materials and methods}

Expression plasmid and cell lines. The QSG7701 human hepatocyte cell line (Department of Cell Biology, Chinese Academy of Science, Shanghai, China) and empty plasmid pcDNA3.0 (Invitrogen, Carlsbad, CA, USA) were stored at our laboratory. The recombinant plasmid, pcDNA-X, containing the $H B V-X$ gene was constructed by The Key Laboratory of Viral Hepatitis (Hunan, China).

Reagents. A reverse transcriptase kit (Fermentas, Waltham, MA USA), a protein extraction kit and an ECL chemiluminescence kit were obtained from Invitrogen; Thermo Fisher, Scientific, Inc. (Waltham, MA, USA). Mouse anti-human HBx monoclonal antibodies were purchased from Abcam (Cambridge, UK). Horseradish peroxidase-conjugated rabbit anti-mouse IgG was purchased from Beijing Zhongshan Golden Bridge Biotechnology (Beijing, China). DNMT3A/3B rabbit anti-human polyclonal antibody and horseradish peroxidase-conjugated goat anti-rabbit IgG were purchased from Santa Cruz Biotechnology, Inc. (Dallas, Texas, USA). An immunohistochemistry PV-6002 kit and DAB color kit were purchased from Beijing Zhongshan Golden Bridge Biotechnology Company. A DNA extraction kit was purchased from Tiangen Biotech Co., Ltd. (Beijing, China). A reverse transcription kit and real-time PCR kit were obtained from Invitrogen, Thermo Fisher Scientific, Inc. Radioimmunoprecipitation assay (RIPA) cell lysates and A protein quantification kit were obtained from KGI Biotechnology Development Co., Ltd. (Nanjing, China). The ECL chemiluminescence kit was obtained from the Biyuntian Company (Beijing, China). Rabbit anti-human SOCS-1 monoclonal antibody was purchased from Santa Cruz Biotechnology, Inc.). Mouse anti-human $\beta$-actin monoclonal antibody was purchased from Abcam. Horseradish peroxidase-conjugated anti-rabbit and mouse goat anti-mouse secondary antibodies were purchased from China Beijing Zhongshan Golden Bridge Company. The methylation kit was purchased from Zymo Research Corporation (Irvine, California, USA).

Primers. Primers were designed according to GenBank (http://www.ncbi.nlm.nih.gov/genbank/), using Primer Premier 5.0 design software (Premier Biosoft, Palo Alto, CA, USA), and were synthesized by Sangon Biotech Co., Ltd. (Shanghai, China) and Beijing Genomics Institute (Shenzhen, China). The following primers were used: HBV-X, sense 5'-AAGGTACCATGCTGCTAGGCTGTGCT-3' and antisense 5'-CTGGGCCCTTAGGCAGAGGTGGAAAAA GTTG-3', with a fragment size of 481 bp; human DNMT3, sense 5'-CACAGAAGCATATCCAGGA-3'; and antisense 5'-CACATTCTCAAAGAGCCAGA-3', with a target fragment size of $181 \mathrm{bp}$; and human DNMT3B, sense 5'-AGTATC AGGATGGGAAGGAG-3' and antisense 5'-CGATAGGAG ACGAGCTTATTG-3', with a target fragment size of $210 \mathrm{bp}$. According to GenBank, and using Primer Premier 5.0 design software the following primer were constructed: $\beta$-actin, sense 5'-CTCCATCCTGGCCTCGCTGT-3' and antisense 5'-GCTGTCACCTTCACCGTTCC-3', with a fragment size of 242 bp; and SOCS-1, sense5'-AGGGAGCGGATGGGTGT-3' and antisense 5'-GGTAGGAGGTGCGAGTTCAG-3', with a target fragment size of $202 \mathrm{bp}$. The SOCS-1 methylated and unmethylated primers were designed according to the literature (10) as follows: Methylated SOCS1, 5'-TTCGCGTGTATT TTTAGGTCGGTC-3' and antisense: 5'-CGACACAACTCC TACAACGACCG-3', with a target fragment size of $160 \mathrm{bp}$; and unmethylated SOCS1, sense 5'-TTATGAGTATTTGTG TGTATTTTTAGGTTGGTT-3' and antisense 5'-CACTAA CAACACAACTCCTACAACAACCA-3' with a target fragment size of $175 \mathrm{bp}$. All primers were synthesized by Sangon Biotech Co., Ltd. (Shanghai, China) and Beijing Genomics Institute (Shenzhen, China).

Cell culture and stable transfection. The QSG7701, pcDNA-X/QSG7701 and pcDNA3.0/QSG7701 cells were cultured at a density of $2 \times 10^{5}$ cells $/ \mathrm{ml}$ and incubated at $37^{\circ} \mathrm{C}$ $\left(5 \% \mathrm{CO}_{2}\right)$, and the culture media was replaced appropriately for digestion and passage. High sugar Dulbecco's modified Eagle's medium (DMEM; Gibco; Thermo Fisher Scientific, Inc.) with $10 \%$ fetal bovine serum (Gibco; Thermo Fisher Scientific, Inc.) was used. The cells were transfected using LIP02000 transfection reagent (Invitrogen; Thermo Fisher Scientific, Inc.), according to the manufacturer's protocol. Briefly, empty recombinant plasmid pcDNA3.0 and HBx-encoding pcDNA-X were transfected into the QSG7701 cells and cultured in medium containing $400 \mu \mathrm{g} / \mathrm{ml} \mathrm{G} 418$ (Geneticin, Gibco; Thermo Fisher Scientific, Inc.) for 2 weeks to obtain stable transfectant cell clones, termed the pcDNA-X/QSG7701 cells and pcDNA3.0/QSG7701 cells.

Reverse transcription-quantitative polymerase chain reaction (RT-qPCR) detection of the mRNA expression of $H B x$ in pcDNA-X/QSG7701 cells. Cells in the logarithmic growth phase were used for the extraction of total RNA using a TRIzol Reagent kit (Invitrogen; Thermo Fisher Scientific, Inc.). The first strand of cDNA was synthesized using $1 \mu \mathrm{g}$ RNA and a reverse transcription kit, according to the manufacturer's instructions. The PCR amplification conditions were as 
follows: $94^{\circ} \mathrm{C}$ denaturation for $3 \mathrm{~min}$, followed by denaturation at $94^{\circ} \mathrm{C}$ for $30 \mathrm{sec}, 68^{\circ} \mathrm{C}$ annealing for $30 \mathrm{sec}, 72^{\circ} \mathrm{C}$ extension for $1 \mathrm{~min}$ in 35 cycles. Finally, the reaction was extended at $72^{\circ} \mathrm{C}$ for an additional $5 \mathrm{~min}$. The amplified products were applied to a $2 \%$ agarose gel (Sigma-Aldrich, St. Louis, MO, USA) and, following $30 \mathrm{~min}$ of $80 \mathrm{~V}$ electrophoresis, the bands were observed with an ultraviolet (UV) analyzer (Bio-Rad Laboratories, Inc., Hercules, CA, USA).

RT-qPCR detection of the mRNA expression of SOCS-1 in pcDNA-X/QSG7701 cells. Cells in the logarithmic growth phase were processed using TRIzol reagent (Invitrogen; Thermo Fisher Scientific, Inc.) to extract total RNA, which was then quantified and reverse transcribed, according to the manufacturer's protocol of the reverse transcription kit, to yield a cDNA template. PCR amplification was as follows: $95^{\circ} \mathrm{C} 30 \mathrm{sec}, 95^{\circ} \mathrm{C}$ for $10 \mathrm{sec}$ and $58^{\circ} \mathrm{C}$ for $30 \mathrm{sec}$, with 40 cycles for each DNA template in triplicate wells. To measure the mRNA expression levels of DNMT3A/3B, the PCR parameters were as follows: $95^{\circ} \mathrm{C}$ for $30 \mathrm{sec}, 95^{\circ} \mathrm{C}$ for $10 \mathrm{sec}$ and $62^{\circ} \mathrm{C}$ for $30 \mathrm{sec}$ for 40 cycles. All experiments were repeated three times.

Western blot analysis to determine the expression of $H B x$ in pcDNA-X/QSG7701 cells. The proteins were purified on ice using a protein extract kit (KGI Biotechnology, Nanjing, China), according to the manufacturer's protocol. The concentration of the proteins was determined using a bicinchoninic acid protein assay kit (KGI Biotechnology). Subsequently, 2X SDS gel loading buffer (Beyotime Institute of Biotechnology, Shanghai, China), containing $50 \mathrm{mmol} \mathrm{L}-1$ Tris $\mathrm{HCl}$ (pH 6.8); $100 \mathrm{mmol} / 1 \mathrm{DTT} ; 2 \% \mathrm{SDS} ; 0.1 \%$ bromophenol blue and $10 \%$ glycerol, was added, following boiling $\left(100^{\circ} \mathrm{C}\right.$ for $\left.3 \mathrm{~min}\right)$. The denatured proteins $(30 \mu \mathrm{g})$ were then separated with SDS-PAGE and transferred onto a nitrocellulose membrane (Sigma-Aldrich), which was immersed in 5\% skim milk, containing $5 \mathrm{~g}$ skim milk and $100 \mathrm{ml}$ phosphate buffered saline (PBS), at room temperature for $1 \mathrm{~h}$. Subsequently, HBX-specific antibodies (cat. no. ab157480; 1:50) were added (1:50) and GAPDH antibody (cat. no. ab181602; 1:2,000) was incubated at $37^{\circ} \mathrm{C}$ on a horizontal shaker for $1 \mathrm{~h}$, followed by incubation at $4^{\circ} \mathrm{C}$ overnight. Following washing with PBS, horseradish peroxidase-conjugated secondary antibodies corresponding to monkey anti-goat IgG (cat. no. ab112764; 1:1,000) and goat anti-mouse IgG (cat. no. ab150115; 1:1,000) were added, respectively at $37^{\circ} \mathrm{C}$ on a horizontal shaker for $2 \mathrm{~h}$. The membrane was then washed thoroughly and enhanced chemiluminescence (Thermo Fisher Scientific, Inc.) was used to detect bands. The film was exposed for 3-5 min, and was then developed and fixed.

Immunocytochemistry detection of the protein expression of $D N M T 3 A / 3 B$. Immunocytochemistry was performed, according to the manufacturer's instructions. Briefly, the cells were cultured as before. The cells were washed twice with PBS at room temperature and were subsequently fixed in $4 \%$ paraformaldehyde for $20 \mathrm{~min}$ at room temperature. The cells were rinsed three times with PBS and could be stored in $0.02 \%(\mathrm{w} / \mathrm{v})$ sodium azide in PBS at $4{ }^{\circ} \mathrm{C}$ for several days. The cells were incubated with $0.1 \%$ Triton $\mathrm{X}-100$ in
PBS for $15 \mathrm{~min}$ at room temperature, and were subsequently rinsed as before. Blocking was performed using serum in PBS for $1 \mathrm{~h}$ at room temperature. The cells were then incubated in the appropriate primary antibody, rabbit anti-human polyclonal anti-DNMT3A/B (cat. no. sc-20703; Santa Cruz Biotechnology, Inc., Santa Cruz, CA, USA; 1:500) either overnight at $4^{\circ} \mathrm{C}$ or $2 \mathrm{~h}$ at room temperature. The cells were rinsed as before and were incubated with secondary antibody (goat anti-rabbit IgG conjugated to HRP; cat. no. sc-2004; Santa Cruz Biotechnology, Inc.) for $2 \mathrm{~h}$ at room temperature. Following incubation, the cells were risned with blocking solution for $10 \mathrm{~min}$ three times in the dark. The cells were subsequently incubated with DAB for $10 \mathrm{~min}$ at room temperature. The coverslips were then mounted onto slides using mounting medium and assessed using a fluorescent microscope. The scoring criteria of the immunohistochemical analysis were based on IPP images. First, the statistically valid areas of interest (AOI) were selected, and the IOD was calculated using the images of each experimental group. The mean \pm standard deviation IOD were analyzed and compared to determine significant differences between the groups.

Methylation specific PCR (MSP) assay to detect SOCS-1 gene promoter methylation. Cells in the logarithmic growth phase $\left(10^{6}\right.$ cells $)$ were collected for DNA extraction (Tiangen Biotech Co., Ltd.). DNA was quantified using UV spectrophotometry, and the purity was evaluated using an OD260/OD280 ratio $(\sim 1.8)$, suggesting high purity. Purity was also assessed using a $1.5 \%$ agarose gel, which revealed one band, indicating a high level of DNA integrity. For each sample, an EZ DNA Methylation-Gold ${ }^{\mathrm{TM}}$ kit (Zymo Research Corporation) was used, according to the manufacturer's protocol. Subsequently, $1 \mu \mathrm{g}$ DNA was treated with sodium bisulfite to induce a $\mathrm{C}-\mathrm{U}$ transition. If $\mathrm{CpG}$ island methylation occurred, the C-U conversion cannot occur. Based on this principle, methylated and unmethylated PCR primers were designed for specific detection of gene methylation (10). The synthesis of primers, primer sequences and amplified fragment sizes are as described above. The modified DNA was used as a template for the PCR reaction. The total volume of the PCR reaction was $50 \mu \mathrm{l}$, comprising $10 \mu \mathrm{l} 5 \mathrm{X}$ buffer, $5 \mu 1$ of $2 \mathrm{mmol} / 1 \mathrm{dNTP}$ Mix, $2 \mu \mathrm{l} 1 \mu \mathrm{mol} / 1$ of each primer, $4 \mu \mathrm{l}$ of $25 \mathrm{mmol} / \mathrm{l}$ magnesium chloride, 0.5 units Taq enzyme and $5 \mu 1$ modified template DNA. The PCR reaction conditions were as follows: $95^{\circ} \mathrm{C}$ for $2 \mathrm{~min}, 95^{\circ} \mathrm{C}$ for $30 \mathrm{sec}, 55^{\circ} \mathrm{C}$ for $30 \mathrm{sec}, 72^{\circ} \mathrm{C}$ for $1 \mathrm{~min}$ and $72^{\circ} \mathrm{C}$ for $7 \mathrm{~min}$. The QSG7701 DNA $\left(10^{6}\right)$ was modified using $\mathrm{CpG}$ methylase to induce the methylation of all $\mathrm{CpG}$ islands. Following methylation, the DNA was modified with bisulfite, as above. The resulting DNA was used as the positive control, and $\mathrm{ddH}_{2} \mathrm{O}$ as the negative control. The positive and negative controls, together with the samples, were then amplified by PCR, as above, and the PCR products were analyzed using $2 \%$ agarose gel electrophoresis and ethidium bromide staining, followed by quantification with a UV gel image analyzer.

Statistical analysis. SPSS 16.0 statistical software (SPSS, Inc., Chicago, IL, USA) was used for statistical analyses. All data are expressed as the mean \pm standard deviation. One-way analysis of variance was used to compare two groups, and the 
Table I. mRNA and protein expression levels of SOCS-1 in transfected QSG7701 cells.

\begin{tabular}{lccr}
\hline Expression & QSG7701 & pcDNA3.0/QSG7701 & pcDNA3.0-X/QSG7701 \\
\hline mRNA & 1 & $1.0543 \pm 0.19370^{\mathrm{a}}$ & $0.3249 \pm 0.05357^{\mathrm{b}, \mathrm{c}}$ \\
Protein & $0.2152 \pm 0.08165$ & $0.1984 \pm 0.04379^{\mathrm{d}}$ & $0.1496 \pm 0.01056^{\mathrm{e}, \mathrm{f}}$ \\
\hline
\end{tabular}

Data are presented as the mean \pm standard deviation. ${ }^{\mathrm{a} P}>0.05$, vs. QSG7701; ${ }^{\mathrm{P}} \mathrm{P}<0.05$, vs. pcDNA3.0/QSG7701; ${ }^{\mathrm{c}} \mathrm{P}<0.05$, vs. QSG7701; ${ }^{\mathrm{d}} \mathrm{t}=0.803 ; \mathrm{P}>0.05$, vs. QSG7701; ${ }^{\mathrm{e}} \mathrm{t}=4.372 ; \mathrm{P}<0.05$, vs. pcDNA3.0/QSG7701; ${ }^{\mathrm{f}} \mathrm{t}=5.129 ; \mathrm{P}<0.05$, vs. QSG7701.

Table II. Relative mRNA expression levels of DNMT3 A and DNMT3B in transfected QSG7701 cells.

\begin{tabular}{lcrr}
\hline & QSG7701 & pcDNA3.0/QSG7701 & pcDNA3.0-X/QSG7701 \\
\hline DNMT3A & 1 & $1.2149 \pm 0.2186640^{\mathrm{a}}$ & $5.75146 \pm 0.9130863^{\mathrm{b}, \mathrm{c}}$ \\
DNMT3B & 1 & $1.3215 \pm 0.5749459^{\mathrm{d}}$ & $3.79436 \pm 1.2268496^{\mathrm{e}, \mathrm{f}}$ \\
\hline
\end{tabular}

Data are presented as the mean \pm standard deviation. ${ }^{\mathrm{a}} \mathrm{P}>0.05$, vs. QSG7701; ${ }^{\mathrm{b}} \mathrm{P}<0.05$, vs. pcDNA3.0/QSG7701; ${ }^{\mathrm{C}} \mathrm{P}<0.05$, vs. QSG7701; ${ }^{\mathrm{d}} \mathrm{P}>0.05$, vs. QSG7701; ${ }^{\mathrm{e}} \mathrm{P}>0.05$, vs. QSG7701 ${ }^{\mathrm{f}} \mathrm{P}>0.05$, vs. pcDNA3.0/QSG7701.

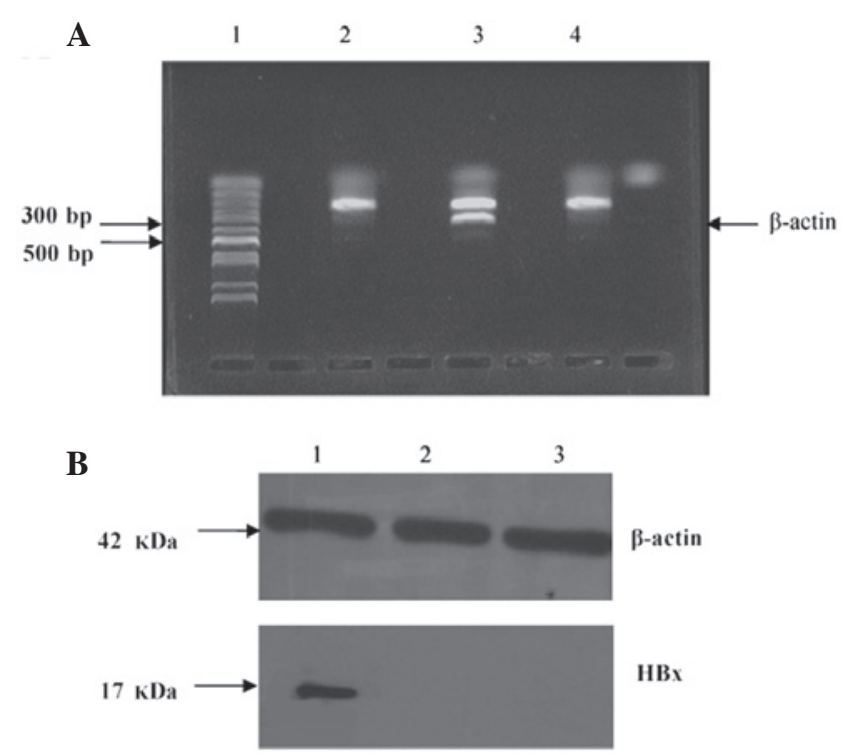

Figure 1. mRNA and protein expression levels of $\mathrm{HBx}$ in transfected QSG7701 cells. (A) Reverse transcription-quantitative polymerase chain reaction detection of the mRNA expression of $\mathrm{HBx}$ in transfected cells. 1 , Marker; 2, QSG7701; 3, pcDNA-X/QSG7701; 4, pcDNA-3.0/QSG7701. (B) Western blot analysis was used to detect the protein expression of $\mathrm{HBx}$ in the transfected cells. 1, pcDNA-X/QSG7701; 2, pcDNA-3.0/QSG7701; 3, QSG7701 cells. HBx, hepatitis B virus X protein.

mean of two samples were compared using the least significant difference $t$-test. $\mathrm{P}<0.05$ was considered to indicate a statistically significant difference.

\section{Results}

mRNA and protein expression of HBx in transfected cells. The total RNA from the pcDNA-X/QSG7701 cells was amplified by RT-qPCR, which generated a band in the region of $500 \mathrm{bp}$, consistent with the expected $481 \mathrm{bp}$. No such band was

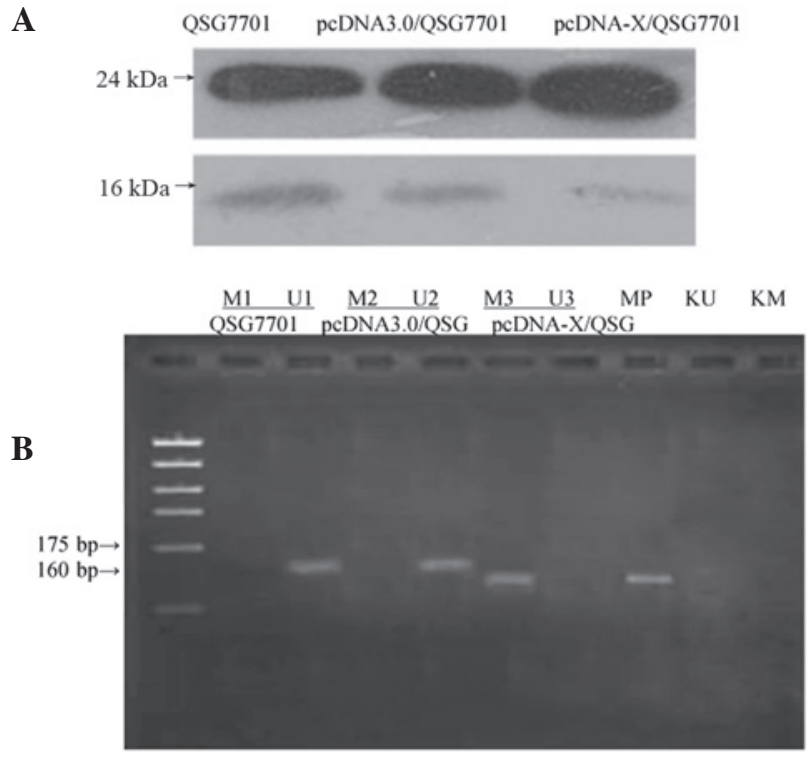

Figure 2. Protein expression and methylation of SOCS-1 in transfected QSG7701 cells. (A) Protein expression of SOCS-1. (B) Methylation of SOCS-1, determined using a methylation-specific polymerase chain reaction assay. M, methylated; U, unmethylated; MP, positive control after treatment with methylase; K, blank control. M1/U1, QSG7701 cells; M2/U2, pcDNA3.0/QSG cells; M3/U3, pcDNA-X/QSG cells. SOCS-1, suppressors of cytokine signaling-1.

identified in the pcDNA3.0/QSG7701 or QSG7701 cell groups, indicating that the mRNA expression of HBV-x was detected in the pcDNA-X/QSG7701 cells (Fig. 1A).

In addition, a specific protein band with a molecular weight of $\sim 17 \mathrm{kD}$ was detected in the pcDNA-X/QSG7701 cells, which is consistent with the expected molecular weight of $\mathrm{HBx}$. No specific bands at this molecular weight were observed in the pcDNA3.0/QSG7701 or SG7701 untransfected cells, indicating that the $\mathrm{HBx}$ protein was expressed in the recombinant pcDNA-X/QSG7701 (Fig. 1B). 
$R T-q P C R$ detection of the MRNA expression of SOCS-1. The mRNA expression of SOCS-1 was significantly lower in the pcDNA-x/QSG7701 cells $(0.3249 \pm 0.05357)$, compared with the pcDNA3.0/QSG7701 cells $(1.0543 \pm 0.19370)$ and QSG7701 cells $(1.0 ; \mathrm{F}=19.6 ; \mathrm{P}<0.05)$. The relative mRNA expression levels of SOCS-1 were marginally higher in the pcDNA3.0/QSG7701 cells (1.0543 \pm 0.19370$)$, compared with the QSG7701 cells (1.0), however, this was not statistically significant $(\mathrm{t}=0.280 ; \mathrm{P}>0.05$; Table $\mathrm{I})$.

Western blotting detection of the protein expression of SOCS-1. The results of the western blot analysis showed that SOCS-1 was expressed in the pcDNA-X-/QSG7701, pcDNA3.0/QSG7701 cells and QSG7701 cells (Fig. 2A). The protein expression levels of SOCS-1 were significantly lower in the pcDNA-X/QSG7701 cells $(0.1496 \pm 0.01056)$, compared with those in the pcDNA3.0/QSG7701 cells $(0.1984 \pm 0.04379)$ and QSG7701 cells $(0.2152 \pm 0.08165 ; \mathrm{F}=19.4 ; \mathrm{P}<0.05)$. The relative protein expression of SOCS-1 was marginally lower in the pcDNA3.0/QSG7701 cells $(0.1984 \pm 0.04379)$, compared with the QSG7701 cells $(0.2152 \pm 0.08165)$, however, this was not statistically significant $(\mathrm{t}=0.803 ; \mathrm{P}>0.05$; Table $\mathrm{I})$.

MSP assay detection of SOCS-1 methylation. The DNA extracted from the transfected cells was modified by bisulfite, and the bisulfited DNA was used as a template for MSP. The primers were methylated or non-methylated SOCS1 primers. The results demonstrated that the PCR reaction with methylated primers resulted in visible bands in the pcDNA-X-/QSG7701 cells, whereas PCR with the unmethylated primers generated no significant bands. In the pcDNA3.0/QSG7701 and QSG7701 cells, a PCR reaction with double distilled water as a template was performed with methylated or unmethylated primers, in which no bands were observed (Fig. 2B).

RT-qPCR detection of $m R N A$ expression levels of DNMT3A and DNMT3B. The relative mRNA expression levels of DNMT3A and DNMT3B were significantly higher in the pcDNA-x/QSG7701 cells, compared with the pcDNA3.0/QSG7701 cells and QSG7701 cells $(\mathrm{P}<0.05)$. The relative mRNA expression levels of DNMT3A and DNMT3B were marginally higher in the pcDNA3.0/QSG7701 cells, compared with the QSG7701 cells, however, this difference was not statistically significant ( $P>0.05$; Table II).

Immunohistochemistry of the protein expression levels of DNMT3A and DNMT3B. Immunohistochemistry was used to measure the protein expression levels of DNMT3A, DNMT3B in the pcDNA-X-/QSG7701, pcDNA3.0/QSG7701 and QSG7701 cells. Positive cells were characterized by brown particles, which appeared inside the cells. The results showed that DNMT3A and DNMT3B were expressed in all three cell groups. Positive signals were predominantly located in the nucleus, and partially in the cytoplasm. No expression was a negative control. Following calculation of the mean IDO of the areas of DNMT3A and DNMT3B protein expression, the expression levels of DNMT3A and DNMT3B in the pcDNA-X/QSG7701 cells were significantly higher, compared with those in the pcDNA3.0/QSG7701 cells and 
A

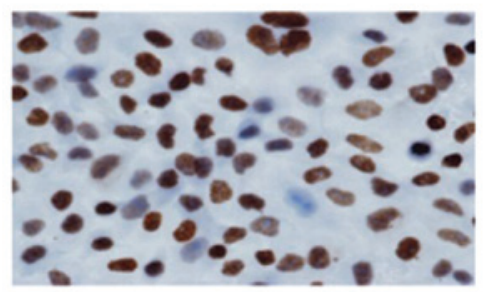

pcDNA-X/QSG7701 ×400

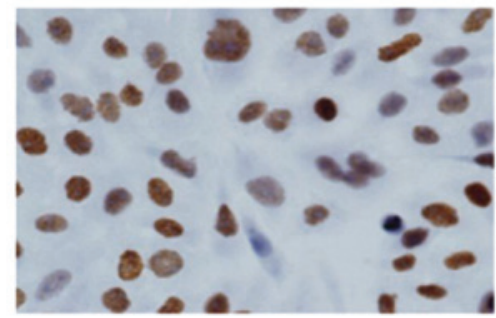

QSG7701 ×400

B

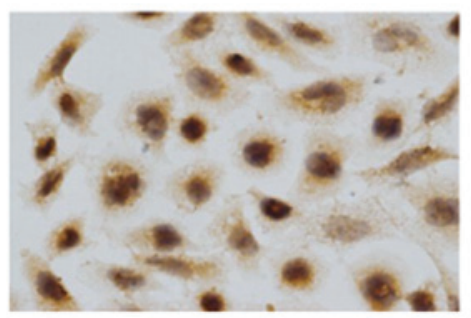

pcDNA-X/QSG7701 ×400

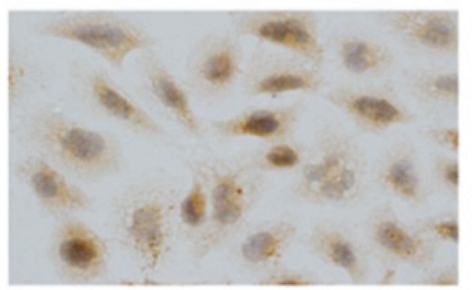

QSG7701 ×400

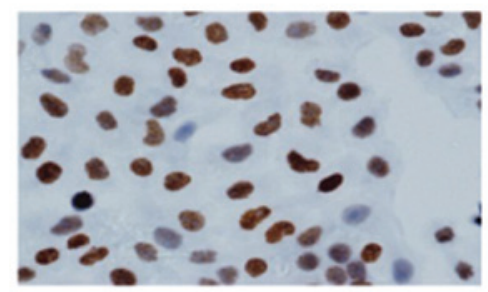

pcDNA3.0/QSG7701 $\times 400$

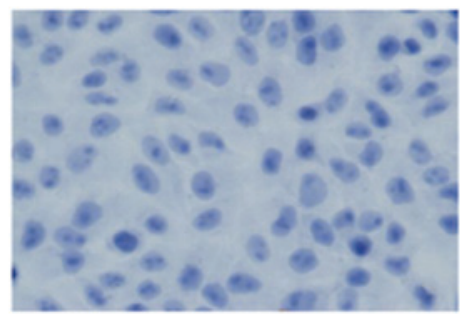

Negative control $\times 400$

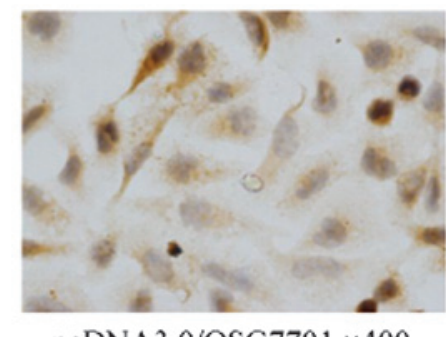

pcDNA3.0/QSG7701 ×400

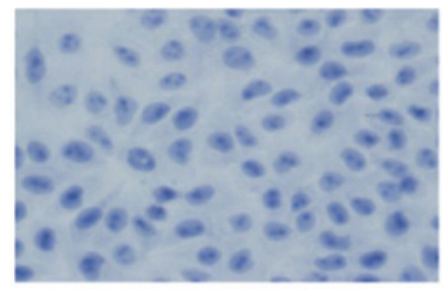

Negative control $\times 400$

Figure 3. Expression of DNMT3A and DNMT3B in transfected QSG7701 cells (magnification, x400). (A) Expression of DNMT3A in the cells. (B) Expression of DNMT3B in the cells. DNMT, DNA methyltransferase.

QSG7701 cells $(\mathrm{P}<0.05)$. The expression levels of these two proteins were marginally higher in the pcDNA3.0/QSG7701 cells, compared with the QSG7701 cells, however, the difference was not statistically significant $(\mathrm{P}>0.05$; Fig. $3 \mathrm{~A}$ and $\mathrm{B}$; Table III).

\section{Discussion}

HBx has been widely recognized as a carcinogen. In addition to its effects on hepatocyte growth, transformation, migration, and apoptosis (11), HBx is also important in HBV-associated hepatocellular carcinoma angiogenesis and metastasis, which may occur through increased expression of vascular endothelial growth factor (VEGF) (12). Previously, HBx was reported to increase heat shock protein $90 \alpha$ (HSP 90 $\alpha$ ), thus enhancing tumor cell invasion (13). Several studies have suggested, that during HBV infection-induced hepatocellular carcinoma, serious disorders of DNA methylation exist in hepatocytes, as well as widespread DNA hypomethylation and local $(\mathrm{CpG}$ island) hypermethylation. The former leads to the abnormal expression of oncogenes, and the latter inactivates several tumor suppressor genes (14-16).

The JAK/STAT signaling pathway is involved in extracellular cytokine signal transduction to the nucleus and, in the liver, cytokines induce the proliferation of hepatocytes by triggering the JAK/STAT signaling pathway. SOCS-1 is an important negative regulatory factor of this JAK-STAT signaling pathway, and previous studies have suggested that SOCS -1 has tumor suppressor activity and may be a potential tumor suppressor gene, exerting negative regulation by inhibiting the expression of certain cancer- or growth-associated genes $(17,18)$. Peng et al reported that SOCS-1 gene CpG island hypermethylation was correlated with gastric cancer development and metastasis (19). Chen et al reported that the intensity of the expression of SOCS-1 in HCC was negatively correlated with pathological grades of cancer. The 1-year-recurrence rate of HCC in SOCS-1 protein-negative patients was higher than that observed in SOCS-1 positive patients, suggesting that 
low expression levels of SOCS-1 are critical to in HCC and metastasis (20). Other studies have indicated that, in patients with HCC and in HCC cell lines, the expression of SOCS-1 is downregulated by gene silencing caused by $\mathrm{CpG}$ island methylation, leading to reduced negative regulation of the JAK/ STAT pathway, which activates the pathway and increases the expression of STAT, particularly STAT3, which is a signaling molecule considered to be associated with abnormal cell proliferation $(3,21,22)$.

The reason for SOCS-1 methylation remains to be elucidated. In addition, whether a causal association exists between the expression of HBx in hepatocytes, SOCS-1 promoter $\mathrm{CpG}$ island methylation and the gene expression of SOCS-1 gene remains to be fully elucidated. Evidence suggests that HBV infection is positively associated with SOCS-1 hepatoma promoter hypermethylation, leading to gene silencing $(23,24)$, which was consistent with the data of the present study. The present study reported that transfection of the QSG7701 cell line with the $H B x$ gene not only promoted SOCS-1 promoter $\mathrm{CpG}$ island methylation, but also reduced the mRNA and protein expression levels of SOCS-1. Previous investigations have resulted in conflicting conclusions. For example, HCC SOCS-1 promoter hypermethylation and reduced expression levels were reported to be unassociated with hepatitis virus infection (21). Ko et al (25) reported that, in 284 liver cancer case specimens, SOCS-1 promoter CpG island hypermethylation was negatively associated with HBSAg-positive cases, but was positively correlated with HCV-Ab-positive cases (26). Such different conclusions are likely to be due to specimen variations.

The $H B V X$-gene encoded HBx protein is a multifunctional regulator with an important regulatory role in apoptosis, cell cycle regulation and cell signal transduction. $\mathrm{HBx}$ is considered to be a viral component closely associated with the development of HCC. Previous studies have show that HBxAg can activate DNA methyltransferase and promote methylation silencing of the cell adhesion-associated cytokine E-cadherin (27) promoter, thereby increasing cell migration activity, as well promoter methylation of the tumor suppressor genes caveolin-1 and p16INK4A $(28,29)$, reducing their expression levels. In the present study, the protein expression of $\mathrm{HBx}$ was upregulated and positively associated with the expression levels of DNMT3A and DNMT3B. It was suggested that $\mathrm{HBx}$ may upregulate the expression of DNMT 3A/3B and promote methylation of the SOCS-1 promoter $\mathrm{CpG}$ island.

DNMTs identified at present include DNMT1, DNMT2, DNMT3A and DNMT3B. Their levels of expression and activity in the colon, stomach, liver, bladder and other tumor tissues are higher than in normal cells, and are key to the re-methylation of certain genes and in maintenance of this methylation status. DNMT3A/3B is important for methylating double-stranded DNA (30), and DNMT3A is responsible for non-CpG island DNA methylation, whereas DNMT3B catalyzes DNA methylation in specific areas, including $\mathrm{CpG}$ islands (31). Park et al (4) reported that HBV X protein not only activates the expression of DNMT1 and DNMT3A, but may also recruit DNMT3A and CpG island methylation connexin 2 to the insulin-like growth factor-binding protein 3 gene promoter region to create a de novo methylation state, inhibiting gene expression. $\mathrm{HBx}$ has also been reported to downregulate the expression of DNMT3B in HCC cell lines, causing overall hypomethylation in hepatoma cells, suggesting that the expression of DNMTS in HCC is key to the development of liver cancer. Aberrant methylation is present in several tumor suppressor genes, including p16 (32), Ras-related protein domain family 1A (33), SOCS1 (21) and glutathione S-transferase pi (34). HBx has been known to increase transcription and translation of DNMT1 and DNMT3AA, and it can induce p16INK4A gene methylation to inhibit the protein expression of p16 protein (29). This suggests that $\mathrm{HBx}$ may inactivate tumor suppressor genes by upregulating methyltransferase and enhancing promoter methylation. Additional investigations have indicated that targeting HBx siRNA and methylation inhibitors can inhibit HCC growth, which may be through a mechanism in which HBx siRNA and methylation inhibitors reverse $\mathrm{HBx}$-induced p16INK4A gene methylation, and thus restore the protein expression and function of p16. In addition, HBx in HCC has been found to be positively associated with methionine adenosyl transferase (MAT)1A and MAT2A, which may be through hypermethylation of the MAT1A promoter and hypomethylation of the MAT2A promoter, leading to the change in the expression status of MATs (35). Jung et al (36) reported that $\mathrm{HBx}$ can increase the expression of the DNMT genes, leading to increased methylation of certain target genes and a reduction intheir functions. A study by Zheng et al (37) suggested that HBx and DNMT3A proteins induce increased methylation of regulatory elements in several genes, leading to transcriptional silencing, and induce hypomethylation in the regulatory elements in several other genes, leading to transcriptional activation of these genes. This may a mechanism for HBx-induced HCC.

In conclusion, the present study provided evidence that transfection of QSG7701 cells with the $H B x$ gene downregulated the expression, and enhanced the methylation, of the SOCS- 1 gene, and upregulated the mRNA and protein expression levels of DNMT3A/B. These results suggest a potential mechanism underlying the $\mathrm{HBx}$-induced development of HCC, which may be through upregulation of the expression of DNMT3A/B, thereby enhancing promoter methylation of the SOCS-1 gene, downregulating the expression of SOCS-1. Downregulated SOCS-1 then leads to the activation of oncogenes. Further investigations are required to verify this potential mechanism.

\section{References}

1. Nguyen VT, Law MG and Dore GJ: Hepatitis B-related hepatocellular carcinoma: Epidemiological characteristics and disease burden. J Viral Hepat 16: 453-463, 2009.

2. Rabe C, Cheng B and Caselmann WH: Molecular mechanisms of hepatitis B virus-associated liver cancer. Dig Dis 19: 279-282, 2001.

3. Yoshikawa H, Matsubara K, Qian GS, Jackson P, Groopman JD, Manning JE, Harris CC and Herman JG: SOCS-1, a negative regulator of the JAK/STAT pathway, is silenced by methylation in human hepatocellular carcinoma and shows growth-suppression activity. Nat Genet 28: 29-35, 2001.

4. Park IY, Sohn BH, Yu E, Suh DJ, Chung YH, Lee JH, Surzycki SJ and Lee YI: Aberrant epigenetic modifications in hepatocarcinogenesis induced by hepatitis B virus X protein. Gastroenterology 132: 1476-1494, 2007.

5. Wang ZG and Wu JX: DNA methyltransferase: Classification, research progress. Yi Chuan 31: 903-912, 2009 (In Chinese). 
6. Jang SJ, Soria JC, Wang L, Hassan KA, Morice RC, Walsh GL, Hong WK and Mao L. Activation of melanoma antigen tumor antigens occurs early in lung carcinogenesis. Cancer Res 61: 7959-7963, 2001

7. Roman-Gomez J, Jimenez-Velasco A, Castillejo JA, Agirre X, Barrios M, Navarro G, Molina FJ, Calasanz MJ, Prosper F, Heiniger A and Torres A: Promoter hypermethylation of cancerrelated genes: A strong independent prognostic factor in acute lymphoblastic leukemia. Blood 104: 2492-2498, 2004

8. Chen L, Zhu Z, Sun X, Dong XY, Wei J, Gu F, Sun YL, Zhou J, Dong JT and Fu L: Down-regulation of tumor suppressor gene FEZ1/LZTS1 in breast carcinoma involvespromoter methylation and associates with metastasis. Breast Cancer Res Treat 116 : 471-478, 2009

9. Mendez N, Orenes-Piñero E, Gaspar M, Fierro M, Ecke T, Gil M, Palou J, et al: Discovery of the methylation of the metastasis suppressor gene, KISS-1, in bladder cancer. J Urology 181: 374 2009.

10. Yu J, Ni M, Xu J, Zhang H, Gao B, Gu J, Chen J, Zhang L, Wu M, Zhen S and Zhu J: Methylation profiling of twenty promoter$\mathrm{CpG}$ islands of genes which may contribute to hepatocellular carcinogenesis. BMC Cancer 2: 29, 2002.

11. Murakami S: Hepatitis B Virus X Protein: Structure, function and biology. Intervirology 42: 81-99, 1999.

12. Zhao H, Liu KG, Shang HL, et al: The correlation of $\mathrm{HBx}$ and VEGF expression with angiogenesis and metastasis in HBV related hepatocelular carcinoma. Chin Hepatol 1: 15-18, 2010.

13. Li W, Miao X, Qi Z, Zeng W, Liang J and Liang Z: Hepatitis $B$ virus $x$ protein upregulates HSP90 alpha expression via activation of c-myc in human hepatocarcinoma cell line, HepG2. Virol J 7: 45, 2010

14. Gui Y, Yeganeh M, Ramanathan S, Leblanc C, Pomerleau V, Ferbeyre G, Saucier C and Ilangumaran S: SOCS-1 controls liver regeneration by regulating HGF signaling in hepatocytes. J Hepatol 55: 1300-1308, 2011.

15. Liu W, Li X, Chu ES, Go MY, Xu L, Zhao G, Li L, Dai N, Si J, Tao Q, Sung JJ and Yu J: Paired box gene 5 is a novel tumor suppressor in hepatocellular carcinoma through interaction with p53 signaling pathway. Hepatology 53: 843-853, 2011.

16. Yoshida T, Ogata H, Kamio M, Joo A, Shiraishi H, Tokunaga Y, Sata M, Nagai $\mathrm{H}$ and Yoshimura A: SOCS1 is a suppressorof liver fibrosis and hepatitis-induced carcinogenesis. J Exp Med 199: 1701-1707, 2004

17. Zhang YJ, Li H, Wu HC, Shen J, Wang L, Yu MW, Lee PH, Bernard Weinstein I, Santella RM: Silencing of Hint1, a novel tumor suppressor gene, by promoter hypermethylation in hepatocellular carcinoma. Cancer Lett 275: 277-284, 2009.

18. Venkitachalam S, Chueh FY, Leong KF, Pabich S and Yu CL: Suppressor of cytokine signaling 1 interacts with oncogenic lymphocyte-specific protein tyrosine kinase. Oncol Rep 25: 677-683, 2011.

19. Peng XC, Ma HY and Wang CQ: Aberrant methylation of suppressor of Ccytokine signaling-1 gene in human gastric carcinomas. Journal of Yangtze University (Natural Science Edition) 5: 5-8, 2008.

20. Chen NP, Jiang YG, Miao H, et al: Expression and clinical significance of SOSC-1 in human hepatocellular cancer. J Exp Surg 2: 845-847, 2008.

21. Chu PY, Yeh CM, Hsu NC, Chang YS, Chang JG and Yeh KT: Epigenetic alteration of the SOCS-1 gene in hepatocellular carcinoma. Swiss Med Wkly 140: w13065, 2010.
22. Tamiya T, Kashiwagi I, Takahashi R, Yasukawa H and Yoshimura A Suppressors of cytokine signaling (SOCS) proteins and JAK/STAT pathways: regulation of T-cell inflammation by SOCS1 and SOCS3. Arterioscler Thromb Vasc Biol 31: 980-985, 2011.

23. Kiran M, Chawla YK and Kaur J: Methylation profiling of tumor suppressor genes and oncogenes in hepatitis virus-related hepatocellular carcinoma in northern India. Cancer Genet Cytogenet 195: 112-119, 2009.

24. Nomoto S, Kinoshita T, Kato K, Otani S, Kasuya H, Takeda S, Kanazumi N, Sugimoto H and Nakao A: Hypermethylation of multiple genes as clonal markers in multicentric hepatocellular carcinoma. Br J Cancer 97: 1260-1265, 2007.

25. Ko E, Kim SJ, Joh JW, Park CK, Park J and Kim DH: CpG island hypermethylation of SOCS-1 gene is inversely associated with HBV infection in hepatocellular carcinoma. Cancer Lett 271: 240-250, 2008

26. Miyoshi H, Fujie H, Shintani Y, Tsutsumi T, Shinzawa S, Makuuchi M, Kokudo N, Matsuura Y, Suzuki T, Miyamura T, et al: Hepatitis $\mathrm{C}$ virus core protein exerts an inhibitory effect on suppressor of cytokine signaling (SOCS)-1 gene expression. J Hepatol 43: 757-763, 2005.

27. Su PF, Lee TC, Lin PJ, Lee PH, Jeng YM, Chen CH, Liang JD, Chiou LL, Huang GT and Lee HS: Differential DNA methylation associated with hepatitis B virus infection in hepatocellular carcinoma. Int J Cancer 121: 1257-1264, 2007.

28. Yan J, Lu Q, Dong J, Li X, Ma K and Cai L: Hepatitis B virus $\mathrm{X}$ protein suppresses caveolin-1 expression in hepatocellular carcinoma by regulating DNA methylation. BMC Cancer 12: 353, 2012.

29. Zhu YZ, Zhu R, Shi LG, Mao Y, Zheng GJ, Chen Q and Zhu HG: Hepatitis $B$ virus $X$ protein promotes hypermethylation of p16(INK4A) promoter through upregulation of DNA methyltransferases in hepatocarcinogenesis. Exp Mol Pathol 89: 268-275, 2010.

30. Liu LM, Luo L, Jing M, et al: HBx in HCC pathogenesis and biological treatment strategies. World J Gastroenterol 13 432-439, 2005.

31. Zhang YG and Liu BC: Functions of DNA methyltransferase and methylation in tumor generation. Int J Surg 35: 537-540, 2008.

32. Zhang JC, Lu J, Li HP, et al: p16 promoter hypermethylation in the plasma DNA and its possible application in molecular diagnosis of hepatocellular carcinoma. Zhonghua Jian Yan Yi Xue Za Zhi 29: 895-898, 2006

33. Schagdarsurengin U, Wikens L, Steinemann D, Flemming P, Kreipe HH, Pfeifer GP, Schlegelberger B and Dammann R: Frequent epigenetic inactivation of the RASSF1A gene in hepatocellular carcinoma. Oncogene 22: 1866-1871, 2003.

34. Yang B, Guo M, Herman JG and Clark DP: Aberrant promoter methylation profiles of tumor suppressor genes in hepatocellular carcinoma. Am J Pathol 163: 1101-1107, 2003.

35. Wang DF, Liu S, Liu QY, et al: The relationship between $\mathrm{HBx}$ and methylation status of MATs promoters in hepatocellular carcinoma. J Exp Surg 28: 242-244, 2011

36. Jung JK, Arora P, Pagano JS and Jang KL: Expression of DNA methytranferase 1 is activated by hepatitis $B$ virus $X$ protein via a regulatory circuit involving the p16INK4a-cyclin D1-CDK 46/ pRb-E2F1 pathway. Cancer Res 67: 5771-5778, 2007.

37. Zheng DL, Zhang L, Cheng N, Xu X, Deng Q, Teng XM, Wang KS, Zhang X, Huang J and Han ZG: Epigenetic modification induced by hepatitis $B$ virus $X$ protein via interaction with de novo DNA methyltransferase DNMT3A. J Hepatol 50: 377-387, 2009. 